\title{
Efficient Analysis of Input Impedance and Mutual Coupling of Microstrip Antennas Mounted on Large Coated Cylinders
}

\author{
V. B. Ertürk, Member, IEEE, and R. G. Rojas, Fellow, IEEE
}

\begin{abstract}
An efficient and accurate hybrid method, based on the combination of the method of moments (MoM) with a special Green's function in the space domain is presented to analyze antennas and array elements conformal to electrically large material coated circular cylinders. The efficiency and accuracy of the method depend strongly on the computation of the Green's function, which is the kernel of the integral equation that is solved via MoM for the unknown equivalent currents representing only the antenna elements. Three types of space-domain Green's function representations are used, each accurate and computationally efficient in a given region of space. Consequently, a computationally optimized analysis tool for conformal microstrip antennas is obtained. Input impedance of various microstrip antennas and mutual coupling between two identical antennas are calculated and compared with published results to assess the accuracy of this hybrid method.
\end{abstract}

Index Terms-Coated cylinders, Green's function, input impedance, method of moments, mutual coupling.

\section{INTRODUCTION}

$\mathbf{T}$ HERE have been major advances in the area of computeraided design (CAD) technology directed toward the development of efficient and accurate numerical methods for the design and analysis of microstrip antennas and arrays. Although there are many practical applications that require the use of antennas that conform to their supporting surfaces, most work on microstrip elements has been for planar structures. There are some techniques that can be used for the analysis of conformal arrays; however, they are usually restricted to small arrays mounted on electrically small cylinders. This necessitates the development of efficient analytical and numerical tools for this class of antennas conformal to electrically large, cylindrically shaped substrates.

Several techniques to design/analyze cylindrical microstrip antennas and arrays have been reported for a number of applications [1]-[10]. Among them, cavity-model analysis [2], [3] and generalized transmission-line model (GTLM) theory [8], [9] are fairly simple and accurate models but not suitable for many structures, in particular, if the thickness of the substrate is not very thin [10]. On the other hand, full-wave solutions [4]-[7] are more accurate and applicable to many structures. The

Manuscript received February 25, 2001; revised July 19, 2001.

V. B. Ertürk is with the Department of Electrical and Electronics Engineering, Bilkent University, TR-06533 Bilkent, Ankara, Turkey.

R. G. Rojas is with the Department of Electrical Engineering, ElectroScience Laboratory, The Ohio State University, Columbus, OH 43212-1191 USA.

Digital Object Identifier 10.1109/TAP.2003.811060 full-wave analysis of microstrip antennas and arrays on coated circular cylinders has been mainly performed using a method of moments (MoM)/Green's function technique in the spectral domain where the traditional eigenfunction series representation of the Green's function is used as the kernel of the integral equation [4]-[6]. However, due to the computational complexity of the solution, which involves a series summation in terms of Bessel and Hankel functions and a Fourier integral, most of the numerical results have been given for microstrip antennas mounted on circular cylinders with electrically small radii. Furthermore, the spectral-domain representation of the Green's function has serious convergence problems for electrically large separations between source and observation points on electrically large cylinders. This makes the analysis of mutual coupling between microstrip antennas intractable, in particular, at high frequencies. Carefully chosen basis functions for the expansion of the patch surface currents can alleviate this problem only to an extent. A few asymptotic representations for the dyadic Green's function of impedance and dielectric coated circular conducting cylinders have been presented to overcome this difficulty [11]-[15]. However, an asymptotic Green's function must be accurate for arbitrary source and observation locations if it is to be used in an MoM-based solution. As shown here, more than one asymptotic Green's function representation may be required for an efficient and accurate analysis of microstrip antennas/arrays mounted on electrically large material-coated circular cylinders. Purely numerical techniques such as the finite-element as well as the finite-difference time-domain [16] techniques are becoming popular and have also been used for the analysis of conformal antennas. Although purely numerical techniques can handle arbitrary geometries, they are not efficient when the structures become electrically large.

In this paper, we present a highly efficient and accurate hybrid method to design/analyze microstrip antennas and arrays conformal to electrically large material coated circular cylinders. This hybrid method is based on the combination of the MoM with a special Green's function in the space domain. To obtain a high degree of accuracy, three different space-domain representations are used for this special Green's function, each chosen on the basis of its computational efficiency and the region where it remains accurate. Consequently, a computationally optimized design/analysis tool for conformal microstrip antennas and arrays is obtained. In Section II, development of the integral equation and expressions for three different space-domain representations are given along with the MoM solution. In Section III, numerical results involving the mutual impedance between two 


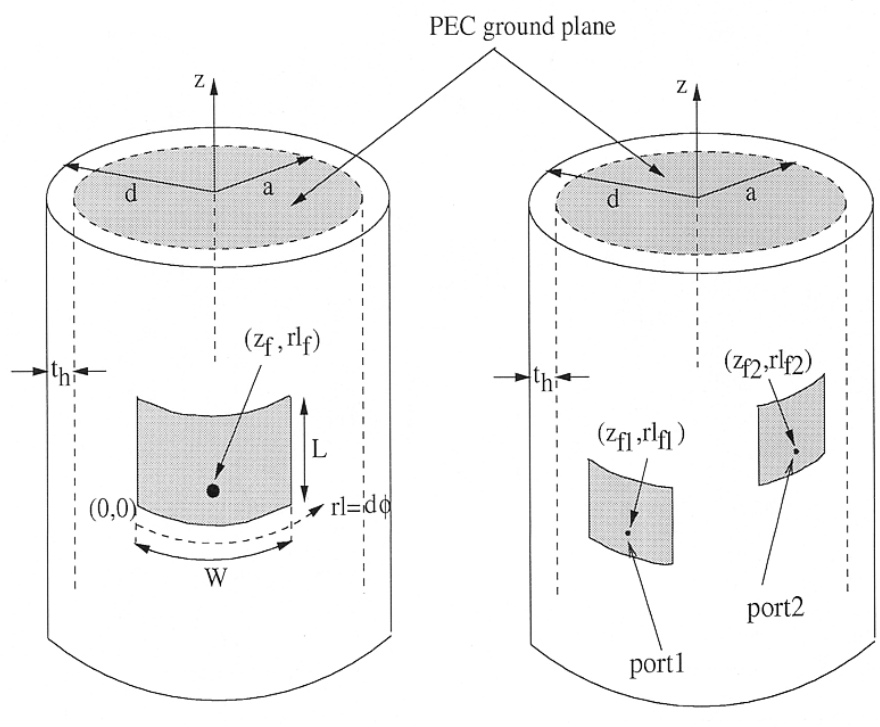

(a)

(b)

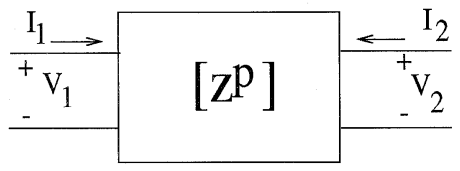

(c)

Fig. 1. Geometry for (a) input impedance and (b) mutual coupling calculations. (c) Definitions of two-port voltages and currents.

current modes, input impedance of various microstrip antennas, and mutual coupling between two identical microstrip antennas are calculated and compared with published results to assess the accuracy of this hybrid method. An $e^{j \omega t}$ time dependence is assumed and suppressed throughout this paper.

\section{FORMULATION}

Fig.1 (a) depicts a probe-fed rectangular microstrip antenna, which will be used to perform the input impedance calculations. Fig.1 (b) illustrates two probe-fed rectangular microstrip antennas used to perform the mutual impedance calculations. In both cases, the antennas are fed at the feed locations $\left(z_{f}, r l_{f}\right)$ and are mounted on the outer surface of a cylindrically shaped dielectric substrate with an inner radius $a$, outer radius $d$, thickness $d-a=t_{h}$, and relative permittivity $\epsilon_{r}>1$. The substrate is backed by a perfectly conducting, cylindrically shaped ground plane. Both the ground plane and cylinder are assumed to be infinite in the $z$-direction.

\section{A. Development of Integral Equation and Green's Function}

Using the Schelkunoff's surface equivalence principle [17], an equivalent problem is obtained where the conducting patches are replaced by equivalent induced currents that are unknown and are to be obtained via MoM. The total electric field on the surface of the substrate can be written as

$$
\mathbf{E}(\mathbf{r})=\mathbf{E}^{i}(\mathbf{r})+\mathbf{E}^{s}(\mathbf{r})
$$

where $\mathbf{E}^{i}(\mathbf{r})$ is the field generated by a known probe current density $\mathbf{J}_{i p}\left(\mathbf{r}^{\prime}\right)$ in the presence of a dielectric coated perfect electric conducting (PEC) circular cylinder and is given by

$$
\mathbf{E}^{i}(\mathbf{r})=\iiint_{V_{\text {source }}} \overline{\mathbf{G}}\left(\mathbf{r}, \mathbf{r}^{\prime}\right) \cdot \mathbf{J}_{i p}\left(\mathbf{r}^{\prime}\right) d v^{\prime} .
$$

Similarly, $\mathbf{E}^{s}(\mathbf{r})$ is the scattered field and is given by

$$
\mathbf{E}^{s}(\mathbf{r})=\iint_{S_{\mathrm{patch}}} \overline{\mathbf{G}}\left(\mathbf{r}, \mathbf{r}^{\prime}\right) \cdot \mathbf{J}^{s}\left(\mathbf{r}^{\prime}\right) d s^{\prime}
$$

where $\mathbf{J}^{s}\left(\mathbf{r}^{\prime}\right)$ is the unknown induced surface current to be determined.

The electric field integral equation (EFIE) is a statement of the boundary condition that the total electric field (1) tangential to the surface covered by the metallic microstrip patch should vanish such that

$$
\hat{n} \times \mathbf{E}(\mathbf{r})=\hat{n} \times\left[\mathbf{E}^{i}(\mathbf{r})+\mathbf{E}^{s}(\mathbf{r})\right]=0 \quad \text { on } \quad S_{\text {patch }} .
$$

Then

$$
\begin{gathered}
\hat{n} \times \iint_{S_{\text {patch }}} \overline{\mathbf{G}}\left(\mathbf{r}, \mathbf{r}^{\prime}\right) \cdot \mathbf{J}^{s}\left(\mathbf{r}^{\prime}\right) d s^{\prime}=-\hat{n} \times \iiint_{V_{\text {source }}} \overline{\mathbf{G}}\left(\mathbf{r}, \mathbf{r}^{\prime}\right) \\
\cdot \mathbf{J}_{i p}\left(\mathbf{r}^{\prime}\right) d v^{\prime}
\end{gathered}
$$

This is the EFIE to be solved via MoM for the unknown currents $\mathbf{J}^{s}\left(\mathbf{r}^{\prime}\right)$. The kernel of this integral equation is the coated cylinder's Green's function denoted by $\overline{\mathbf{G}}\left(\mathbf{r}, \mathbf{r}^{\prime}\right)$, where the primed coordinates represent the source location and the unprimed coordinates represent the observation point. 
This Green's function is efficiently calculated for arbitrary source and observation locations using three different types of space-domain representations, each valid and accurate in a different region of space.

The first one is a steepest descent path (SDP) representation of the dyadic Green's function [14]. It is based on a circumferentially propagating ( $\phi$-propagating) series representation of the appropriate Green's function and its efficient numerical evaluation along an SDP on which the integrand decays most rapidly. Using this representation, the surface field component in the $l$-direction at $\rho=d$ excited by a $u$-directed source (again at $\rho^{\prime}=d$ ) is written as [14]

$$
E_{l}(\alpha, s) \approx \frac{\sqrt{2} e^{\frac{-j 3 \pi}{4}}}{4 \pi^{2} d} \sum_{q=1}^{Q} F\left(\alpha, s, t_{q}\right) w_{q}
$$

with

$$
F\left(\alpha, s, t_{q}\right)=\frac{e^{-j k_{0} s}}{\sqrt{k_{o} s-\frac{j t_{q}^{2}}{2}}} \int_{C_{\tau}\left(t_{q}\right)} G_{l u}\left(t_{q}, \tau\right) P_{e}^{u} e^{-j \xi \tau} d \tau
$$

where $s$ is the arc length of the geodesic path on the surface of the coating from the source to the observation locations, $\alpha$ is the angle between the ray path and the circumferential axis, $t_{q}$ are the roots of the Hermite polynomials, and $w_{q}$ are the appropriate weights (Gauss-Hermite quadrature) whose values can be found in [18]. The parameter $\xi=\left(k_{t 0} d / 2\right)^{1 / 3}\left(\phi-\phi^{\prime}\right)$, where $k_{t 0}$ is the transverse propagation constant in free-space and $k_{0}$ is the free-space wave number. The definition of the contour $C_{\tau}\left(t_{q}\right)$ and the explicit expressions for $G_{l u}\left(t_{q}, \tau\right)$ are given in [14]. This representation is valid away from the paraxial (near axial) region and cannot be used for the self-impedance calculations.

The second representation is the paraxial space-domain representation of the dyadic Green's function [15], [19], which complements the SDP representation along the paraxial region. In this formulation, the $\phi$-propagating series representation of the Green's function is periodic in one of its variables and hence can be approximated by a Fourier series (FS), where the coefficients of this series expansion can be easily obtained by a simple numerical integration algorithm. Based on numerical experimentation, it appears that only the two leading terms of the expansion are necessary in most cases. Furthermore, the accuracy of the Green's function as well as the ease of its evaluation are determined by the type of algorithm used to calculate the FS coefficients. Using this representation, the surface fields are given by

$$
\begin{aligned}
E_{z z}(\delta, s) \approx & \frac{-Z_{0}}{2 \pi k_{0}}\left\{k_{0}^{2} P(s)+\frac{\partial^{2}}{\partial z^{2}}[P(s)-Q(s)]\right\} \\
E_{\phi z}(\delta, s) \approx & \frac{-Z_{0}}{2 \pi k_{0}} \frac{\partial^{2}}{\partial z \partial r_{l}}\{M(s)-R(s)\} \\
E_{\phi \phi}(\delta, s) \approx & \frac{-Z_{0}}{2 \pi k_{0}}\left\{k_{0}^{2} U(s)+\frac{\partial^{2}}{\partial r_{l}^{2}}\left[U(s)-\frac{\epsilon_{r}-1}{\epsilon_{r}} W(s)\right]\right\} \\
& +\frac{j Z_{0}}{4 \pi k_{0}}\left\{S(s)-\frac{\partial^{2}}{\partial r_{l}^{2}} T(s)\right\}
\end{aligned}
$$

where $P(s), Q(s), M(s), R(s), U(s), W(s), S(s)$, and $T(s)$ are explicitly given in [15] and [19]. This representation is valid along the paraxial region but is not accurate away from the paraxial region and cannot be used for the self-impedance evaluations. It should be noted that (8)-(10) contain partial derivatives with respect to the coordinates (i.e., with respect to $z$ and $r_{l}=d\left(\phi-\phi^{\prime}\right)$ ). These derivatives can be transferred to the basis and testing functions using integration by parts.

Finally, when the distance $s$ between the source and observation points is small, the Green's function for the cylinder is approximated by the Green's function of a cylinder with an infinite radius (planar approximation). This approximation is accurate for large cylinders because the surface can be considered to be locally flat when $s$ is small. Therefore, a highly efficient integral representation of the planar microstrip dyadic Green's function derived by Barkeshli et al. [20] is used.

It should be mentioned at this point that these different space-domain representations of the dyadic Green's function are more efficient than the standard spectral-domain Green's function representation. In particular, as the electrical size (radius) of the cylinder gets larger, the difference in the elapsed time for the evaluation of each representation strongly favors the space-domain representations. Some examples are given in the numerical results section to illustrate this point.

\section{B. Method of Moments Solution}

The conventional MoM procedure starts with an expansion of $\mathbf{J}^{s}(\mathbf{r})$ in terms of a finite set of subsectional basis functions

$$
\mathbf{J}^{s}(\mathbf{r})=\sum_{n=1}^{N} a_{n} \mathbf{J}_{n}(\mathbf{r})
$$

where

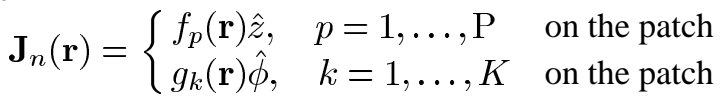

with $N=P+K, a_{n}$ being the unknowns to be computed and

$$
\mathbf{J}_{n}(\mathbf{r}) \neq 0 \quad \text { only if } \mathbf{r} \in S_{n} ; \quad \bigcup_{n=1}^{N} S_{n}=S_{\text {patch }}
$$

The basis functions are selected to be piecewise sinusoidal along the direction of the current and constant in the direction perpendicular to the current. Substituting (12) and (11) into (5), one obtains a single vector equation with $N$ unknowns. Testing this equation by the same set of basis functions (testing functions), which was used in the expansion of the patch current (Galerkin method) and denoted by $\mathbf{w}_{m}(\mathbf{r})(m=1, \ldots, N)$, the following matrix equation is obtained:

$$
[Z] I=V
$$

where

$$
\begin{aligned}
Z_{m n} & =\iint_{S_{m}} d s \mathbf{w}_{m}(\mathbf{r}) \cdot\left(\hat{n} \times \iint_{S_{n}} d s^{\prime} \overline{\mathbf{G}}\left(\mathbf{r}, \mathbf{r}^{\prime}\right) \cdot \mathbf{J}_{n}\left(\mathbf{r}^{\prime}\right)\right) \\
\mathbf{I} & =\left[a_{1}, a_{2}, a_{3}, \ldots, a_{N}\right]^{T} \\
V_{m} & =-\iint_{S_{m}} d s \mathbf{w}_{m}(\mathbf{r}) \cdot \hat{n} \times \mathbf{E}^{i}(\mathbf{r})
\end{aligned}
$$




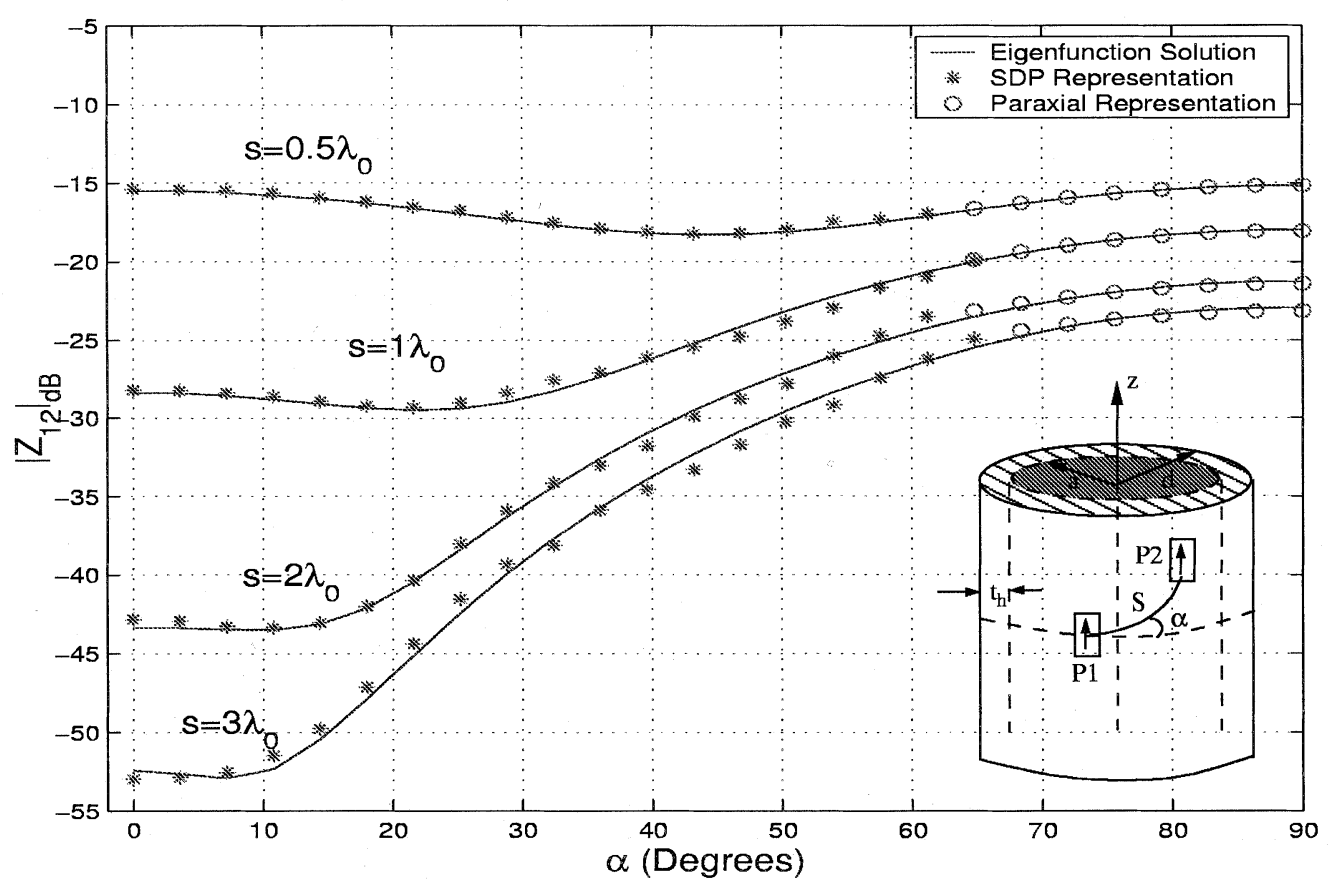

Fig. 2. Mutual impedance $\left(Z_{12}\right)$ between two identical $z$-directed current modes for a coated cylinder with $a=3 \lambda_{0}, t_{h}=0.06 \lambda_{0}, \epsilon_{r}=3.25$.

where $m, n=1, \ldots, N$, and $T$ stands for the transpose operation. This matrix equation can be solved using direct solution methods such as Gaussian elimination, LU-decomposition, or iterative schemes like the conjugate gradient method, including various recently developed acceleration schemes. Once the matrix equation is solved, the induced current can be reconstructed via (11). The calculation of the matrix elements $Z_{m n}$ implies that the Green's function has to be evaluated for arbitrary source and observation points. This requires the development of an algorithm to switch among the three representations of the Green's function. It turns out that the parameter $\xi_{\mathrm{SDP}} / s$ (evaluated at the saddle point) can be used to determine the switching boundary between the SDP and paraxial representations. For $\xi_{\mathrm{SDP}} / s>0.2$ and $s>0.2 \lambda_{0}\left(\lambda_{0}\right.$ is the free-space wavelength), the SDP representation can be used. For $\xi_{\mathrm{SDP}} / s \leq 0.2$ and $s>0.2 \lambda_{0}$, the paraxial representation yields accurate results. Finally, for $s \leq 0.2 \lambda_{0}$, which is necessary for the calculation of the self-impedance as well as the coupling between the adjacent basis functions, the Green's function for the cylinder is approximated by the Green's function of a cylinder of infinite radius (planar approximation).

\section{NUMERICAL RESULTS AND DISCUSSIONS}

The first set of numerical results will illustrate the mutual impedance between two current modes as defined in (15). Results will be given as a function of $\alpha$ for various separations between the source and observation points. Note that (15) can be rewritten in a simpler form, namely

$$
Z_{n m}=\int_{S_{m}} \mathbf{E}_{n} \cdot \mathbf{J}_{m} d s
$$

where $\mathbf{E}_{n}$ is the field due to source $\mathbf{J}_{n}$ and $S_{m}$ is the area occupied by the source $\mathbf{J}_{m}$. These results are presented to assess the accuracy of the switching algorithm (between SDP and paraxial representations) introduced above. In Fig. 2, the calculated mutual impedance between two identical $z$-directed current modes is shown where the SDP and the paraxial representations are combined and the result is compared with the eigenfunction solution for a cylinder with $a=3 \lambda_{0}, t_{h}=0.06 \lambda_{0}$, and $\epsilon_{r}=3.25$. As seen from the figure, the SDP and the paraxial representations complement each other and yield good agreement with the eigenfunction solution. As expected, the coupling is stronger at $\alpha=90^{\circ}$ and weaker at $\alpha=0^{\circ}$. Note also that the results approach the planar case as $s$ becomes smaller. A similar result is illustrated in Fig. 3 for the mutual impedance between $\phi$ and $z$-directed current modes for the same cylinder. The reason the coupling is weak at $\alpha=0, \pi / 2$ is because the crosspolar component of the surface field exhibits a $\sin 2 \alpha$ type pattern, which is similar to the planar case as shown in [21]. Finally, the calculated mutual impedance between two $\phi$-directed current modes mounted on the same cylinder is compared with the eigenfunction solution in Fig. 4. In Figs. 2-4, the computation of the SDP representation is approximately ten times faster than the standard spectral-domain Green's function representation, whereas the paraxial representation is approximately five times faster than the standard spectral-domain Green's function representation.

The next set of numerical results that will be presented are MoM-based results where the input impedance of a probe-fed microstrip antenna and the mutual coupling between two identical microstrip patches on a large coated circular cylinder are calculated.

Let us first consider the two antenna configuration in Fig. 1 (b). The antennas are fed at the feed locations $\left(z_{f_{i}}, r l_{f_{i}}\right)$, 


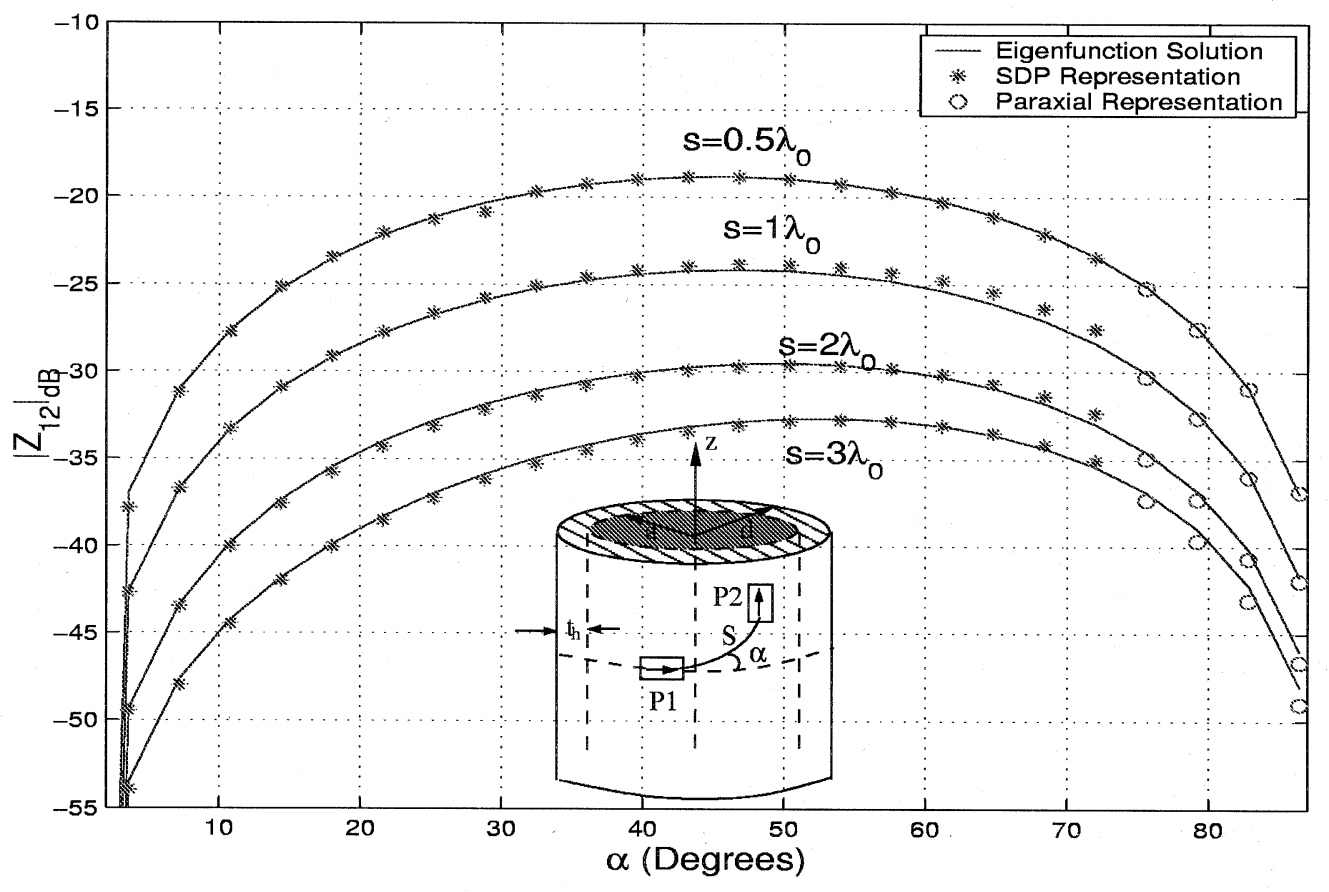

Fig. 3. Mutual impedance $\left(Z_{12}\right)$ between $\phi$ and $z$-directed current modes for a coated cylinder with $a=3 \lambda_{0}, t_{h}=0.06 \lambda_{0}, \epsilon_{r}=3.25$.

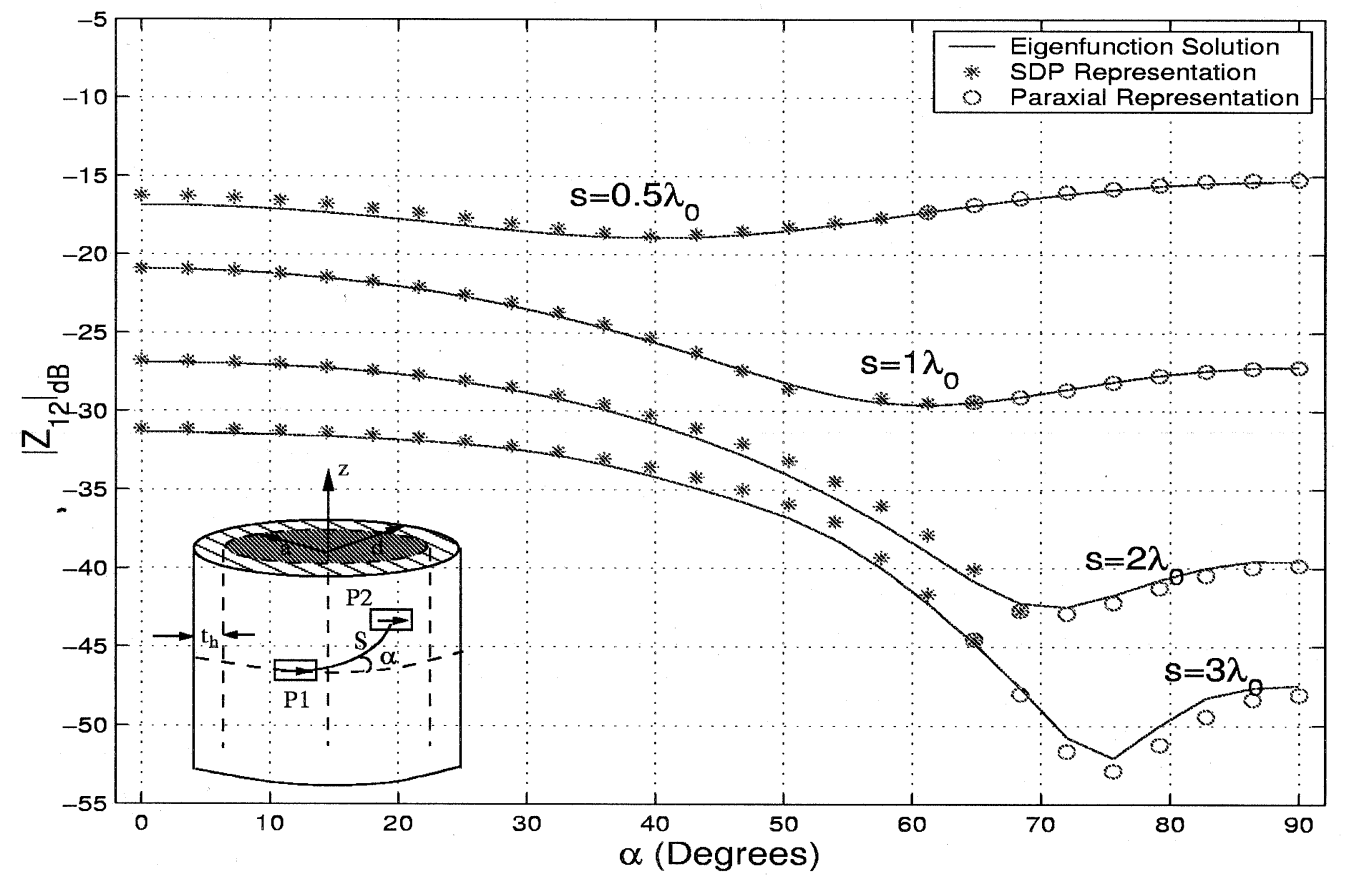

Fig. 4. Mutual impedance $\left(Z_{12}\right)$ between two $\phi$-directed current modes for a coated cylinder with $a=3 \lambda_{0}, t_{h}=0.06 \lambda_{0}, \epsilon_{r}=3.25$.

where $i=1$ denotes the first port and $i=2$ denotes the second one. Based on the two-port configuration of Fig.1 (c), we can write

$$
\begin{aligned}
& V_{1}=Z_{11}^{p} I_{1}+Z_{12}^{p} I_{2} \\
& V_{2}=Z_{21}^{p} I_{1}+Z_{22}^{p} I_{2}
\end{aligned}
$$

where

$$
\begin{aligned}
& Z_{11}^{p}=\left.\frac{V_{1}}{I_{1}}\right|_{I_{2}=0}, Z_{22}^{p}=\left.\frac{V_{2}}{I_{2}}\right|_{I_{1}=0}, \\
& Z_{12}^{p}=\left.\frac{V_{1}}{I_{2}}\right|_{I_{1}=0}, \quad Z_{21}^{p}=\left.\frac{V_{2}}{I_{1}}\right|_{I_{2}=0} .
\end{aligned}
$$




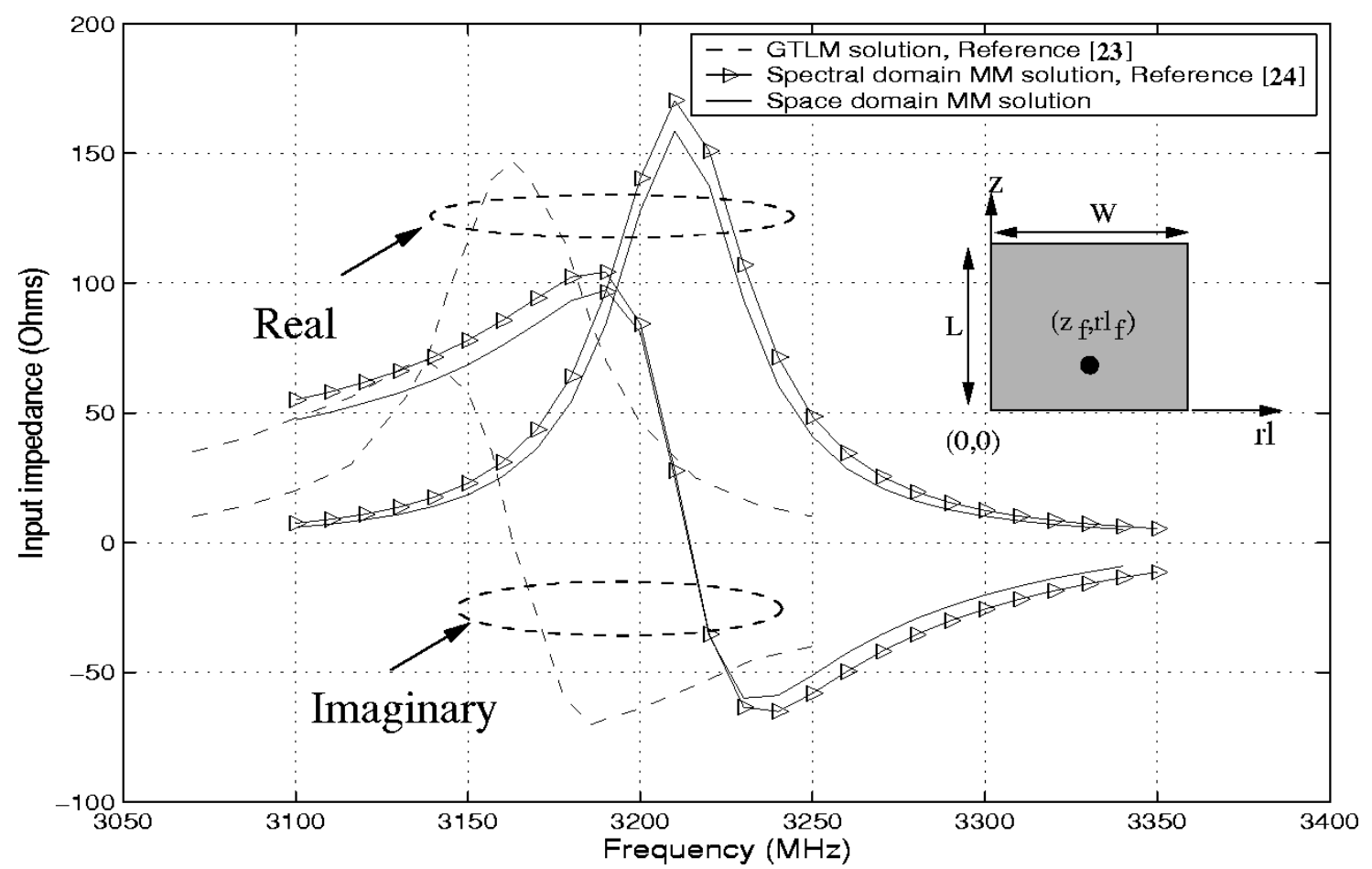

Fig. 5. Input impedance versus frequency for a patch fed via a probe with the following parameters: $a=20 \mathrm{~cm}, \epsilon_{r}=2.32, t_{h}=0.795 \mathrm{~mm}, L=3 \mathrm{~cm}$, $W=4 \mathrm{~cm}$, and $\left(z_{f}, r l_{f}\right)=(0.5 \mathrm{~cm}, 2 \mathrm{~cm})$.

$Z_{11}^{p}$ is the input impedance of element one, with element two open-circuited (the superscript $p$ stands for port), and can be written as [22]

$$
Z_{11}^{p}=\frac{-\int_{v} \mathbf{E}^{(1)} \cdot \mathbf{J}_{i p}^{(1)} d v}{\left(I_{i p}\right)^{2}}
$$

where $\mathbf{E}^{(1)}$ is the total electric field at port one (with port two open) due to the impressed current density $\mathbf{J}_{i p}^{(1)}$ at port one with a terminal current $I_{i p}=1 A$. The use of (3) then gives

$$
Z_{11}^{p}=-\sum_{n=1}^{2 N} a_{n} V_{n}^{(1)}
$$

where $a_{n}$ are the expansion mode coefficients found from (14) and $V_{n}^{(1)}$ are the induced voltages at port one due to the current modes $J_{n}$ in both patches. A similar expression can be used to calculate $Z_{22}^{p}$.

The mutual impedance between ports one and two $Z_{21}^{p}$ can be written as [22]

$$
Z_{21}^{p}=\frac{-\int_{v} \mathbf{E}^{(1)} \cdot \mathbf{J}_{i p}^{(2)} d v}{\left(I_{i p}\right)^{2}}
$$

where $\mathbf{E}^{(1)}$ is the total electric field, at port two, induced by the impressed current $\mathbf{J}_{i p}^{(1)}$ at port one (with port two open) and $\mathbf{J}_{i p}^{(2)}$ is an impressed current source at port two. Note that both impressed sources have terminal currents $I_{i p}=1 A$. Using (3) yields

$$
Z_{21}^{p}=-\sum_{n=1}^{2 N} a_{n} V_{n}^{(2)}
$$

where $V_{n}^{(2)}$ are the induced voltages at port two due to the currents $J_{n}$ in both patches. It should be kept in mind that $Z_{21}^{p}=$ $Z_{12}^{p}$ as a result of the reciprocity theorem. Furthermore, the input impedance for the single antenna depicted in Fig.1 (a) can still be calculated using the expression given by (23); however, the second antenna is removed and not included in the solution of the integral equation. This also implies that $2 N$ becomes $N$ in (23). Note that the two-port parameters $Z_{i j}^{p}$ introduced above should not be confused with (18)

The first numerical example is given for a single rectangular microstrip patch mounted on a dielectric coated circular cylinder with $a=20 \mathrm{~cm}, \epsilon_{r}=2.32, t_{h}=0.795 \mathrm{~mm}$. The length of the patch $L$ is $3 \mathrm{~cm}$, and the width of the patch $W$ is $4 \mathrm{~cm}$. The antenna is excited with a $\mathrm{TM}_{01}$ mode. Fig. 5 shows the input impedance (real and imaginary part) of this cylindrical-rectangular antenna versus frequency where the proposed space-domain MoM result is compared with the result given in [23], which is obtained using the GTLM and with an MoM solution based on a standard spectral-domain Green's function [24]. To compare the space-domain MoM solution with the results given in [23] and [24], the antenna is fed via a probe at the feed location $\left(z_{f}, r l_{f}\right)=(0.5 \mathrm{~cm}, 2 \mathrm{~cm})$, as seen in Fig. 5, and the self-inductance of the probe is accounted by adding $j X_{p r}$ to the input impedance $\left[Z_{i n}=Z_{11}^{p}+j X_{p r}\right.$, where $Z_{11}^{p}$ is given by (23)], as explained in [22], where

$$
X_{p r}=\frac{Z_{0}}{\sqrt{\epsilon_{r}}} \tan \left(\sqrt{\epsilon_{r}} k_{0} t_{h}\right)
$$

and $Z_{0}$ is the characteristic impedance of the feeding coaxial cable (assumed to be $50 \Omega$ here). This is a reasonable approximation for a large cylinder where the thickness of the substrate is thin. The agreement between the space- and spectral-domain 


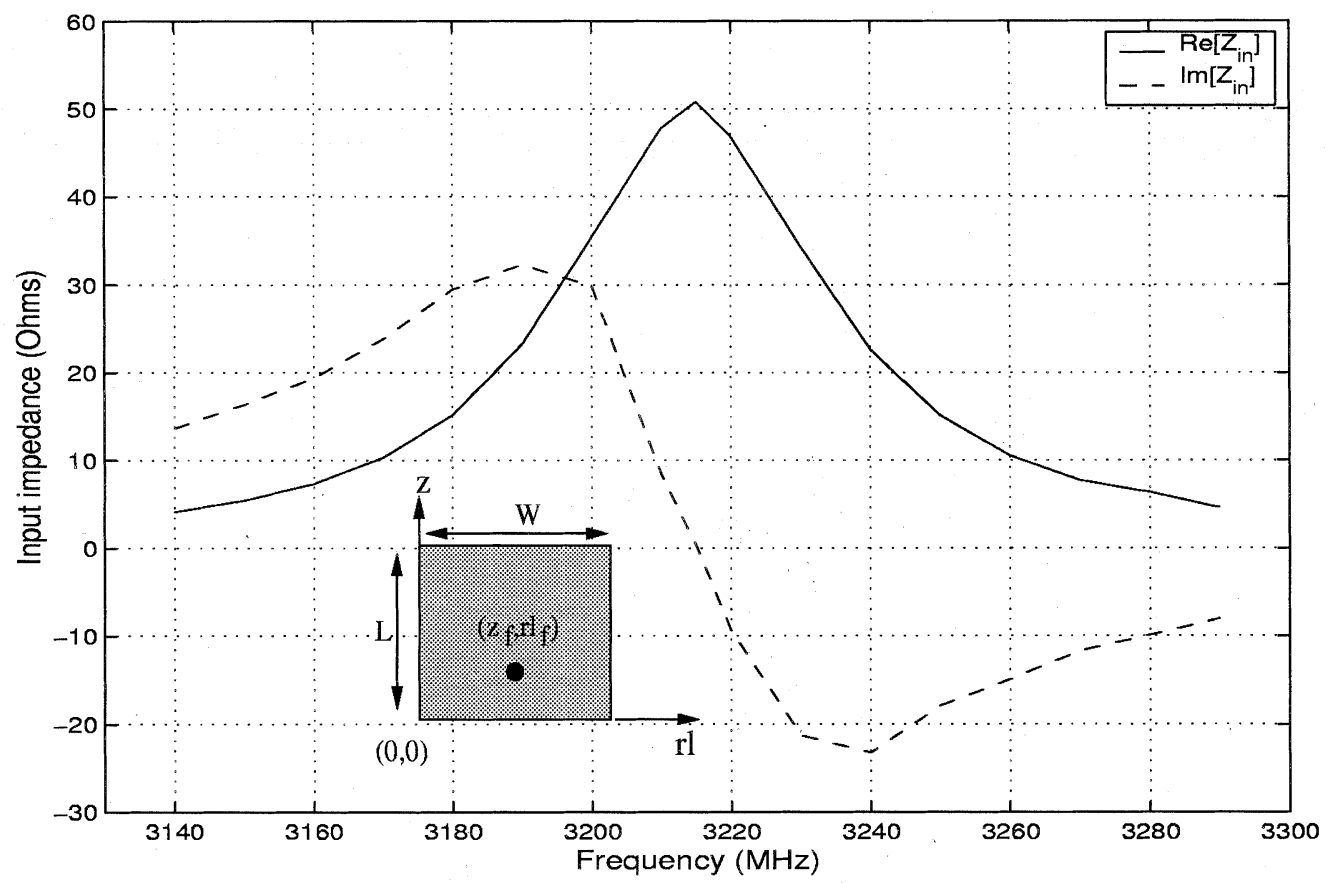

Fig. 6. Input impedance versus frequency for the patch fed via a probe with the following properties: $a=20 \mathrm{~cm}, \epsilon_{r}=2.32, t_{h}=0.795 \mathrm{~mm}, L=3 \mathrm{~cm}$, $W=4 \mathrm{~cm}$, and $\left(z_{f}, r l_{f}\right)=(0.95 \mathrm{~cm}, 2 \mathrm{~cm})$.

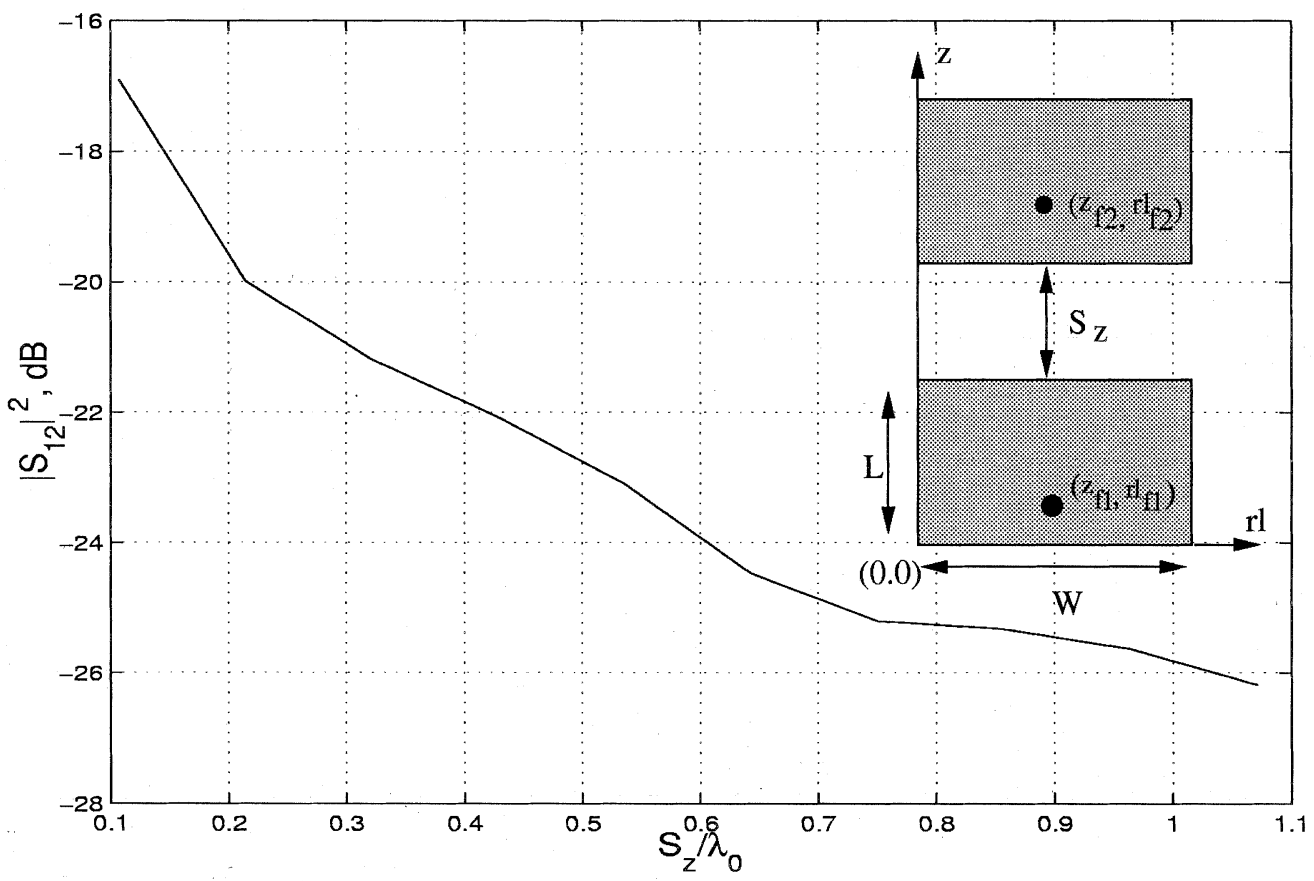

Fig. 7. Mutual coupling coefficient $S_{12}$ for the E plane coupling case at $3215 \mathrm{MHz}$. The antenna parameters are the same as given in Fig. 5 .

results is very good. On the other hand, although the overall agreement is good between the full-wave solutions (space- and spectral-domain MoM solutions) and the GTLM, there is a small frequency shift (less than 1.65\%). It should be kept in mind that the GTLM is an approximate method and some errors are to be expected.

To carry out mutual coupling calculations between two microstrip antennas, it is first necessary to find a feed location that yields an input impedance of $50 \Omega$. If we use the same antenna as in Fig. 5, it turns out that placing the probe at $\left(z_{f}, r l_{f}\right)=$ $(0.95 \mathrm{~cm}, 2 \mathrm{~cm})$ results in a $50-\Omega$ input impedance at the resonance frequency $f \approx 3215 \mathrm{MHz}$, as depicted in Fig. 6. Mutual coupling results can now be obtained for two antennas mounted on the same cylinder and identical to the antenna in Fig. 6. The $\mathrm{E}$ plane and $\mathrm{H}$ plane mutual coupling coefficients versus the edge-to-edge spacing $S$ between them are presented in Figs. 7 


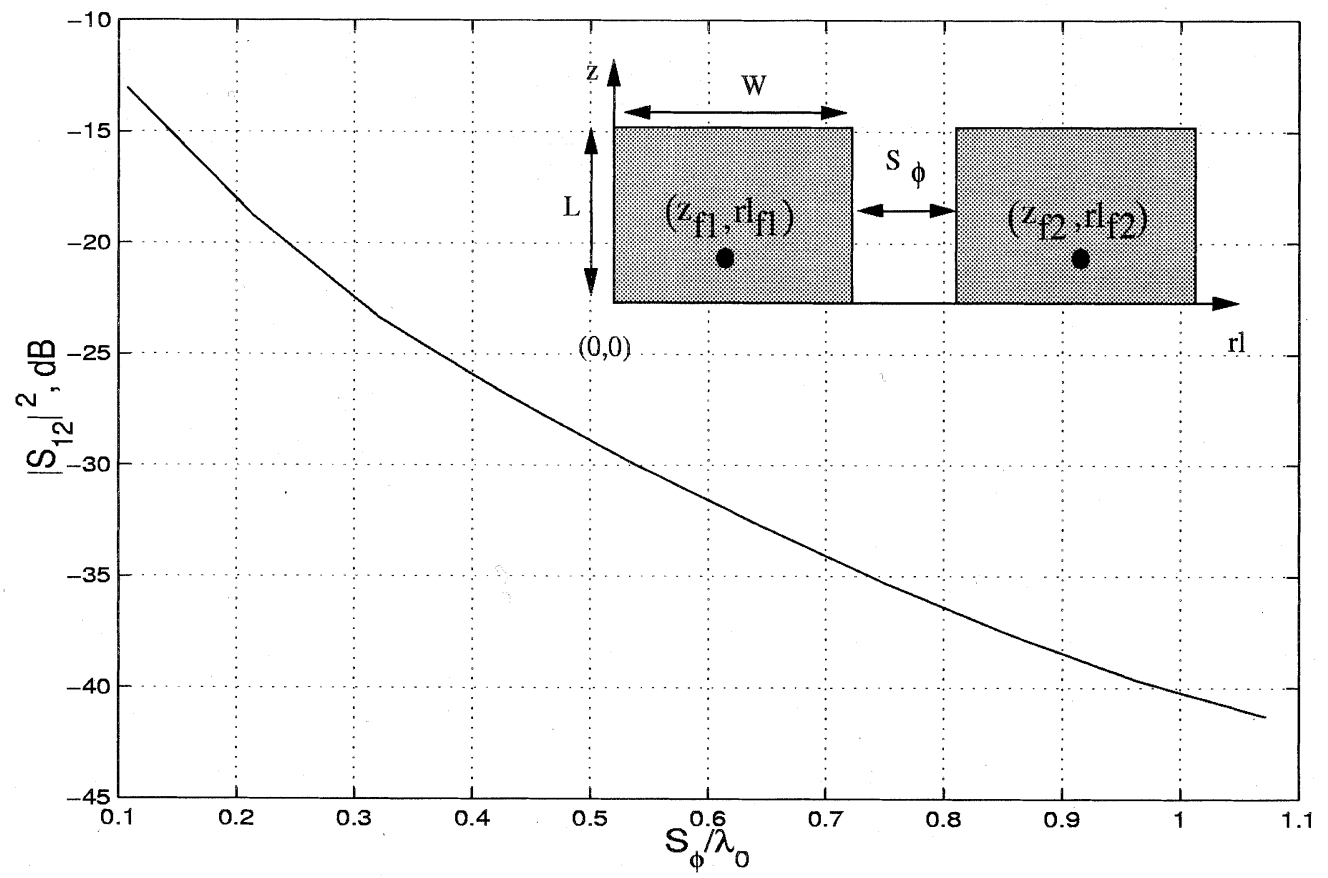

Fig. 8. Mutual coupling coefficient $S_{12}$ for the $\mathrm{H}$ plane coupling case at $3215 \mathrm{MHz}$. The antenna parameters are the same as given in Fig. 5 .

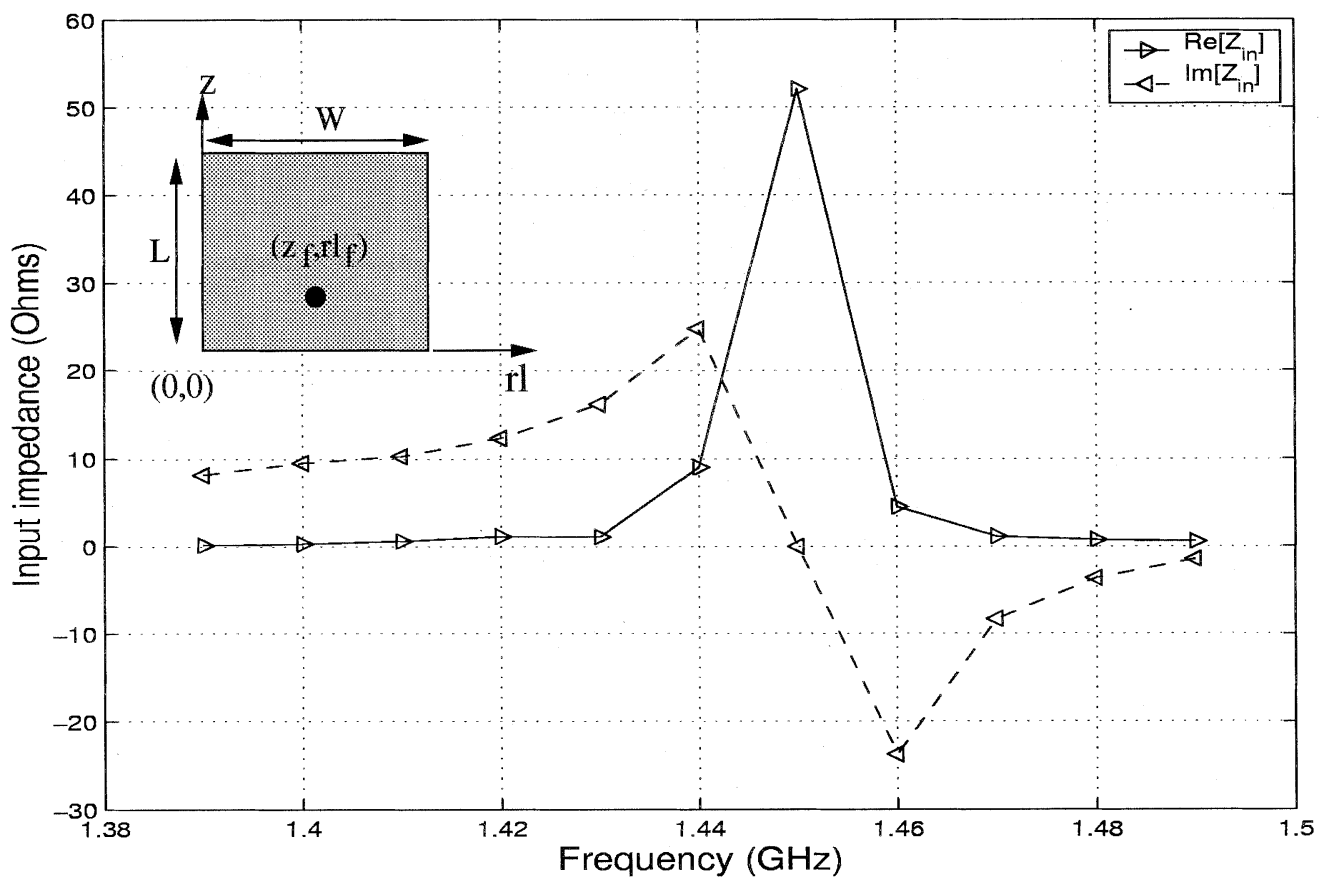

Fig. 9. Input impedance versus frequency for the patch fed via a probe. The antenna parameters are $a=40 \mathrm{~cm}, \epsilon_{r}=2.98, t_{h}=0.762 \mathrm{~mm}, L=6 \mathrm{~cm}$, $W=4 \mathrm{~cm}$

and 8, respectively. In Figs. 7 and 8, the edge-to-edge spacing is normalized with respect to the free-space wavelength $\lambda_{0}$. The operating frequency is $3215 \mathrm{MHz}$. Note that the mutual coupling coefficient can be calculated from [9]

$$
S_{12}=\frac{2 Z_{12}^{p} Z_{0}}{\left(Z_{11}^{p}+Z_{0}\right)\left(Z_{22}^{p}+Z_{0}\right)-Z_{12}^{p} Z_{21}^{p}}
$$

In all these results related to this example, each basis function has the approximate dimensions of 0.15 by $0.075 \lambda_{0}$ at the highest frequency simulated. As a result, the number of basis functions $N$ is 12 [six basis functions along the $z$-direction $(P=6)$ and six basis functions along the $\phi$-direction $(K=6)$ ] for the single antenna case and $N=24$ for the two-antenna case. 


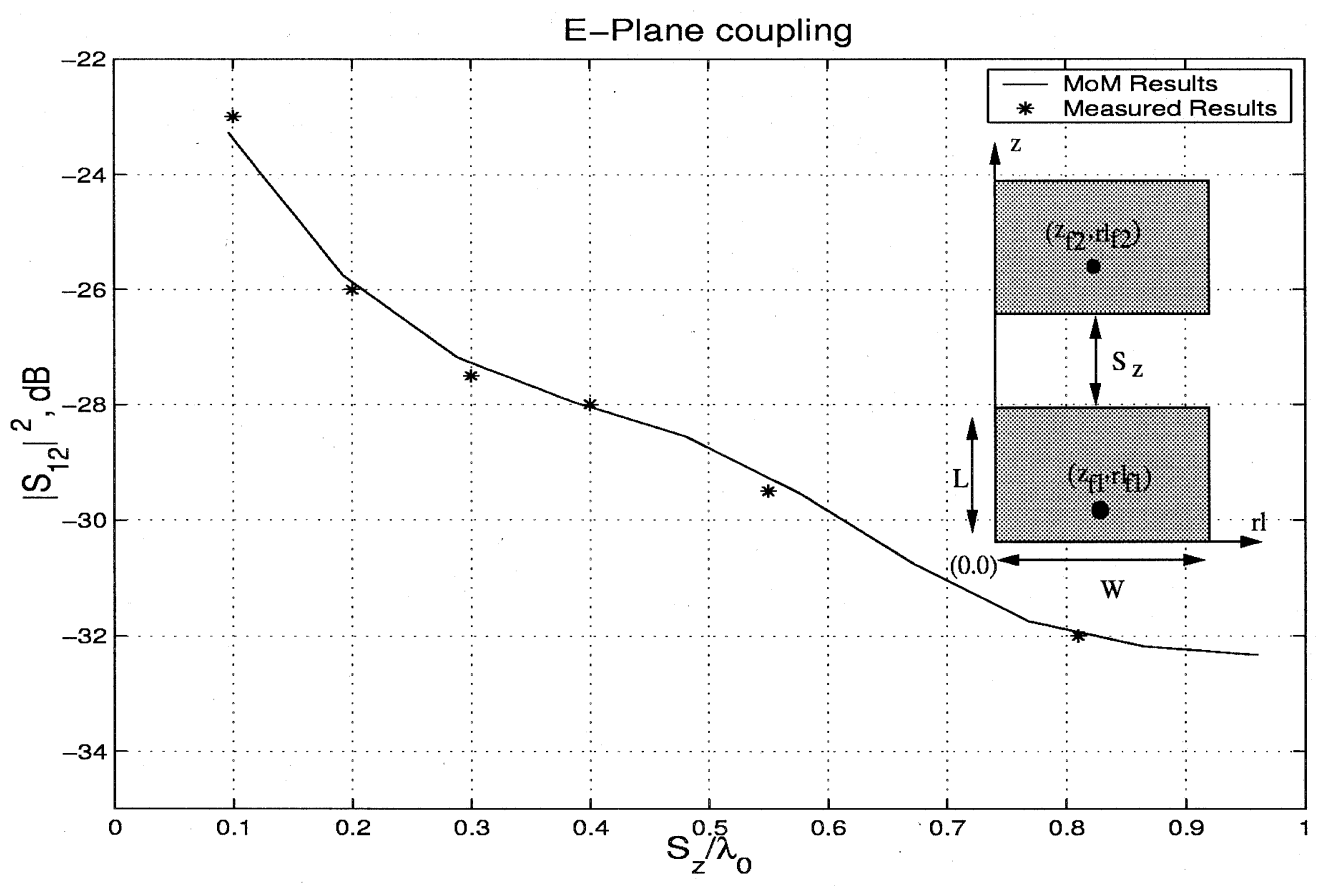

Fig. 10. Mutual coupling coefficient $S_{12}$ for the E plane coupling case at $1.45 \mathrm{GHz}$. The antenna parameters are the same as given in Fig. 9 .

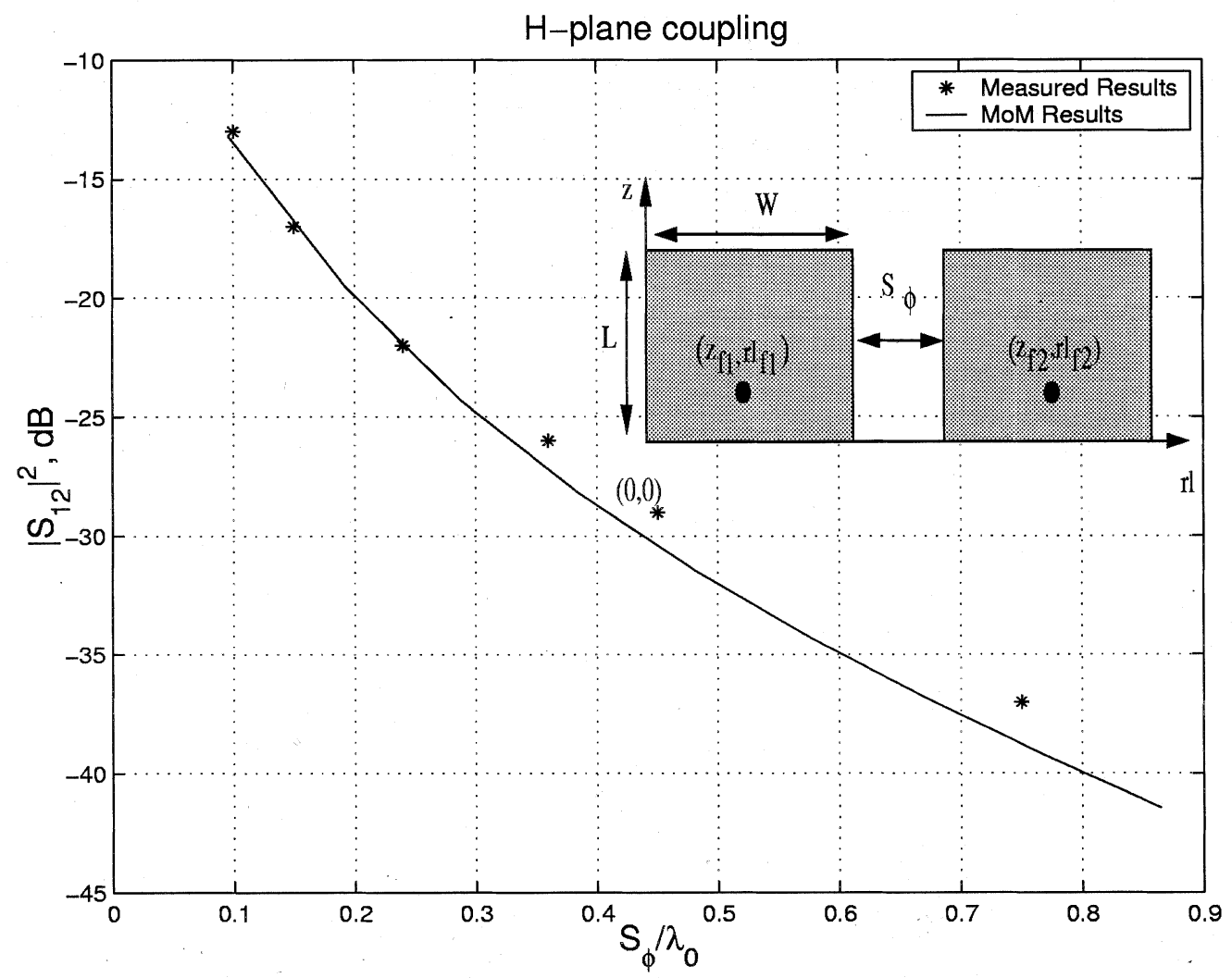

Fig. 11. Mutual coupling coefficient $S_{12}$ for the $\mathrm{H}$ plane coupling case at $1.45 \mathrm{GHz}$. The antenna parameters are the same as given in Fig. 9.

As a second numerical example, the mutual coupling coefficients versus the edge-to-edge spacing $S$ between two identical patches are calculated with the current method and compared with the measured results given in [9] for a cylinder with the following parameters: $a=40 \mathrm{~cm}, \epsilon_{r}=2.98$, and $t_{h}=0.762 \mathrm{~mm}$. The dimensions of the patches are $L=6 \mathrm{~cm}$, 
$W=4 \mathrm{~cm}$, yielding a resonance at $1.45 \mathrm{GHz}$ where the $\mathrm{TM}_{01}$ mode is excited. As in the previous example, the feed position is first adjusted until one obtains a $50-\Omega$ input impedance for each isolated patch. The input impedance (real and imaginary parts) versus frequency of this antenna at the $50-\Omega$ feed location $\left(z_{f}, r l_{f}\right)=(2.1 \mathrm{~cm}, 2 \mathrm{~cm})$ is given in Fig. 9. The mutual coupling (versus distance) between these two identical antennas is calculated in the E plane and $\mathrm{H}$ plane as shown in Figs. 10 and 11 , respectively.

Keeping in mind that the dominant currents for the microstrip antennas considered here are in the direction of the axis of the cylinder, the surface waves excited within the dielectric layer are stronger along the $z$ axis. They also have a slower rate of decay than the space waves, which are the dominant contributors along the $\mathrm{H}$ plane. Consequently, the rate of decay of the $\mathrm{H}$ plane coupling versus distance is larger than the corresponding E plane case. A similar phenomenon is also observed in the planar case [22].

In the above example, each basis function has the approximate dimensions of 0.1 by $0.05 \lambda_{0}$ at the highest frequency simulated. Consequently, the number of basis functions for a single patch is 38 [20 basis functions along the $z$-direction $(P=20)$ and 18 basis functions along the $\phi$-direction $(K=18)]$. Therefore, $N=38$ for Fig. 9, whereas $N=76$ for Figs. 10 and 11 . In both cases, the agreement between measured and calculated results is very good. These results verify the accuracy of the solutions developed in this paper

\section{CONCLUSION}

An efficient and accurate hybrid method based on the combination of the MoM with a special Green's function in the space domain is presented to analyze antennas and array elements conformal to material-coated electrically large circular cylinders. The efficiency and accuracy of the method are strongly dependent on the computation of the Green's function, which is the kernel of the EFIE integral equation. The well-known MoM is used to solve the integral equation to obtain the equivalent surface currents induced on the microstrip patches. The Green's function has to be valid for arbitrary source and observation point locations to calculate the MoM impedance matrix. Therefore, three types of space-domain Green's function representations are used, where each representation is computationally efficient and highly accurate in a given region of space. Fortunately, the three regions overlap with each other and make the switch from one representation to another smooth. The SDP and paraxial representations are used for separations between source and observation points larger than $0.2 \lambda_{0}$. For separations less than $0.2 \lambda_{0}$, one can assume for electrically large cylinders that the region is locally flat. Thus, a planar microstrip Green's function is used to calculate the diagonal as well as near-the-diagonal elements of the impedance matrix. These switching points may have to be slightly adjusted, depending on the degree of accuracy that is required.

The input impedance of various microstrip antennas and mutual coupling between two identical antennas have been calculated and compared with published results to assess the accuracy of this hybrid method. The accuracy of the results was shown to be excellent, thus validating the analysis provided in this paper.

\section{REFERENCES}

[1] S. B. Fonseca and A. J. Giarola, "Analysis of microstrip wraparound antennas using dyadic Green's functions," IEEE Trans. Antennas Propagat., vol. AP-31, pp. 248-253, Mar. 1983.

[2] C. M. Krowne, "Cylindrical-rectangular microstrip antennas," IEEE Trans. Antennas Propagat., vol. AP-31, pp. 194-199, Jan. 1983.

[3] K. M. Luk, K. F. Lee, and J. S. Dahele, "Analysis of cylindrical-rectangular patch antenna," IEEE Trans. Antennas Propagat., vol. 37, pp. 143-147, Feb. 1989.

[4] A. Nakatini, N. G. Alexopoulus, N. K. Uzunoglu, and P. L. E. Uslenghi, "Accurate Green's function computation for printed circuit antennas on cylindrical antennas," Electromagnetics, vol. 6, pp. 243-254, July-Sept. 1986.

[5] T. M. Habashy, S. M. Ali, and J. A. Kong, "Input impedance and radiation pattern of cylindrical-rectangular and wraparound microstrip antennas," IEEE Trans. Antennas Propagat., vol. 38, pp. 722-731, May 1990.

[6] W. Y. Tam, A. K. Y. Lai, and K. M. Luk, "Mutual coupling between cylindrical-rectangular microstrip antennas," IEEE Trans. Antennas Propagat., vol. 43, pp. 897-899, Aug. 1995.

[7] R. C. Hall and D. I. Wu, "Modeling and design of circularly-polarized cylindrical wraparound microstrip antenna," in IEEE Antennas Propagat. Symp. Dig., vol. 1, July 1996, pp. 672-675.

[8] C.-Y. Huang, Y.-H. Liu, and K. L. Wong, "Input impedance calculation of cylindrical-rectangular microstrip antennas using GTLM theory," in IEEE Antennas Propagat. Symp. Dig., vol. 4, June 1995, pp. 1792-1795.

[9] C.-Y. Huang and Y.-T. Chang, "Curvature effects on the mutual coupling of cylindrical-rectangular microstrip antennas," Electron. Lett., vol. 33, pp. 1108-1109, June 1997.

[10] K.-L. Wong, Design of Nonplanar Microstrip Antennas and Transmission Lines. New York: Wiley, 1999.

[11] L. W. Pearson, "A construction of the fields radiated by a z-directed point sources of current in the presence of a cylindrically layered obstacle," Radio Sci., vol. 21, pp. 559-569, July-Aug. 1986.

[12] R. J. Pogorzelski, "On the high-frequency asymptotic evaluation of the potentials of elemental sources on an anisotropic impedance cylinder," Radio Sci., vol. 31, pp. 389-399, Mar.-Apr. 1996.

[13] C. Demirdag and R. G. Rojas, "Mutual coupling calculations on a dielectric coated PEC cylinder using UTD-based Green's function," in IEEE Antennas Propagat. Symp. Dig., vol. 3, Canada, July 1997, pp. $1525-1528$.

[14] V. B. Ertürk and R. G. Rojas, "Efficient computation of surface fields excited on a dielectric coated circular cylinder," IEEE Trans. Antennas Propagat., vol. 48, pp. 1507-1516, Oct. 2000.

[15] _ - "Paraxial space-domain formulation for surface fields on dielectric coated circular cylinder," IEEE Trans. Antennas Propagat., vol. 50, pp. 1577-1587, Nov. 2002.

[16] J. Byun, B. Lee, and F. J. Harackiewicz, "FDTD analysis of mutual coupling between microstrip patch antennas on curved surfaces," in IEEE Antennas Propagat. Symp. Dig., July 1999.

[17] R. F. Harrington, Time-Harmonic Electromagnetic Fields. New York: McGraw-Hill, 1961.

[18] M. Abramowitz and I. A. Stegun, Handbook of Mathematical Functions. New York: Dover, 1970.

[19] V. B. Ertürk, "Efficient hybrid MoM/Green's function technique to analyze conformal microstrip antennas and arrays," Ph.D. dissertation, Dept. of Electrical Engineering, The Ohio State University, 2000.

[20] S. Barkeshli, P. H. Pathak, and M. Marin, "An asymptotic closed-form microstrip surface Green's function for the efficient moment method analysis of mutual coupling in microstrip antennas," IEEE Trans. Antennas Propagat., vol. 38, pp. 1374-1383, Sept. 1990.

[21] M. A. Marin and P. H. Pathak, "An asymptotic closed-form representation for the grounded double-layer surface Green's function," IEEE Trans. Antennas Propagat., vol. 40, pp. 1357-1366, Nov. 1992.

[22] D. M. Pozar, "Input impedance and mutual coupling of rectangular microstrip antennas," IEEE Trans. Antennas Propagat., vol. AP-30, pp. 1191-1196, Nov. 1982.

[23] K. L. Wong, Y. H. Liu, and C. Y. Huang, "Generalized transmission line model for cylindrical-rectangular microstrip antenna," Microwave Opt. Technol. Lett., vol. 7, pp. 729-732, Nov. 1994.

[24] K. W. Lee, private communication, Jan. 2000. 
V. B. Ertürk (M'00) received the B.S. degree in electrical engineering from the Middle East Technical University, Ankara, Turkey, in 1993, and the M.S. and Ph.D. degrees from The Ohio State University (ODU), Columbus, in 1996 and 2000, respectively.

He is currently an Assistant Professor with the Department of Electrical and Electronics Engineering, Blikent University, Ankara. His research interests include design and analysis of planar and conformal arrays as well as active integrated antennas.

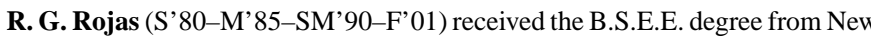
Mexico State University, Las Cruces, in 1979, and the M.S. and Ph.D. degrees in electrical engineering from The Ohio State University, (OSU) Columbus, in 1981 and 1985 , respectively.

He is currently a Professor in the Department of Electrical Engineering with The Ohio State University. His current research interests include the analysis and design of conformal arrays, active integrated arrays, nonlinear microwave circuits, as well as the analysis of electromagnetic radiation and scattering phenomena in complex environments.

Dr. Rojas won the 1988 R.W.P. King Prize Paper Award, the 1990 Browder J. Thompson Memorial Prize Award, both given by IEEE, and the 1989 and 1993 Lumley Research Awards, given by the College of Engineering, OSU. He has served as Chairman, Vice-Chairman, and Secretary/Treasurer of the Columbus, OH Chapter of the IEEE Antennas and Propagation and Microwave Theory and Techniques Societies. He is a Member of U.S. Commission B of URSI. 